\title{
A model for dark current characterization and simulation
}

\author{
Richard L. Baer \\ Agilent Laboratories, 3500 Deer Creek Road, Palo Alto, CA USA 94304
}

\begin{abstract}
The Poisson and Normal probability distributions poorly match the dark current histogram of a typical image sensor. The histogram has only positive values, and is positively skewed (with a long tail). The Normal distribution is symmetric (and possesses negative values), while the Poisson distribution is discrete. Image sensor characterization and simulation would benefit from a different distribution function, which matches the experimental observations better.

Dark current fixed pattern noise is caused by discrete randomly-distributed charge generation centers. If these centers shared a common charge-generation rate, and were distributed uniformly, the Poisson distribution would result. The fact that it does not indicates that the generation rates vary, a spatially non-uniform amplification is applied to the centers, or that the spatial distribution of centers is non-uniform. Monte Carlo simulations have been used to examine these hypotheses.

The Log-Normal, Gamma and Inverse Gamma distributions have been evaluated as empirical models for characterization and simulation. These models can accurately match the histograms of specific image sensors. They can also be used to synthesize the dark current images required in the development of image processing algorithms. Simulation methods can be used to create synthetic images with more complicated distributions.
\end{abstract}

Keywords: dark current, image sensor, probability distribution, histogram, log-normal

\section{Introduction}

A great deal of effort has been expended to reduce the dark current levels in CCD and CMOS image sensors, and to mitigate its effects ${ }^{1,2}$. Dark current creates a spatially-random and temporally-fixed noise pattern that limits the ultimate sensitivity of an imaging system. Because the pattern is fixed and does not vary from frame to frame, it must be associated with irregularities in the pixel. It is generally understood to originate from surface defects at the $\mathrm{SiO} / \mathrm{Si}$ interface and bulk defects in the silicon that introduce mid-band traps which provide a mechanism for thermally-excited carriers to transition between the valance and conduction bands ${ }^{3,4}$. This paper is concerned with the simulation and characterization of this kind of random FPN dark current. The constant dark current background associated with diffusion current and the fixed row and column patterns that are created by imperfect referencing and by column processing circuits are not considered.

Dark current is usually described only by its second order statistics, and frequently just by its mean value ${ }^{5,6}$. Such simple descriptions are misleading because they contain the implicit presumption that the probability distribution is Gaussian. In fact, the Gaussian distribution poorly describes the dark current of a modern image sensor, as shown in Figure 1. Experimentally observed dark current distributions have a significant positive skew. The skew causes a dark current image to appear like the starry night sky, rather than the snowy screen of a TV set tuned to an empty channel. 


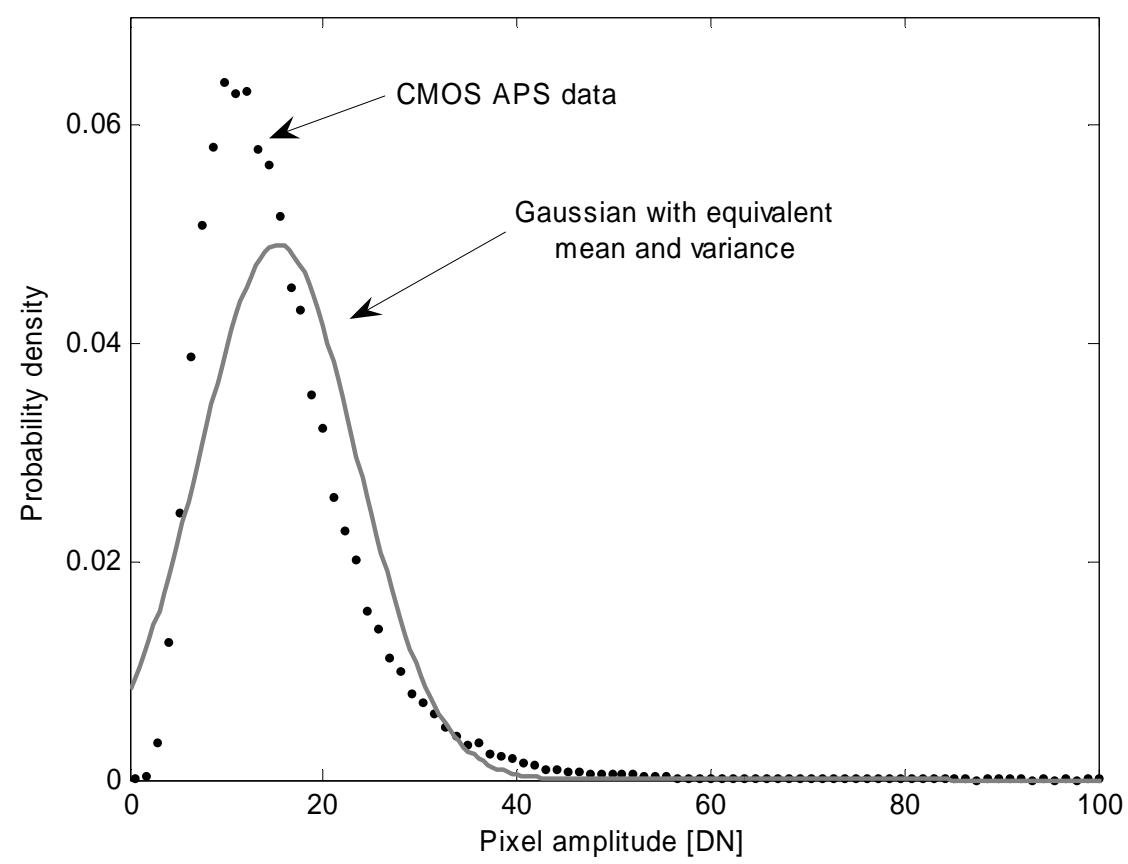

Figure 1: CMOS APS 3T-pixel dark current histogram compared to the Normal distribution with the same $2^{\text {nd }}$ order statistics

Because dark current is associated with randomly distributed defects, use of the Poisson distribution might seem appropriate. However, the Poisson distribution is discrete and its positive skew is only visible when the defect density (and therefore the discreteness of the distribution) is most visible. Computer simulations are used in section two of the paper to determine which physical mechanisms might yield distributions with the correct shape. It is concluded that some sort of spatial non-uniformity is required to match the experimental data.

Although the simulation models are useful for probing the mechanisms behind dark current, they do not lead to analytical solutions that are easy to apply to characterization and simulation. Characterization methods are needed that can describe both the mean value and shape of the distribution with a few parameters. Simulation methods are needed that can produce realistic-looking synthetic dark current images for the development and testing of image processing algorithms. Consequently a number of common distributions with significant positive skew are considered as empirical models in the third section of the paper. The Log-Normal distribution is shown to provide an effective model for a 3T APS CMOS sensor with moderately high dark current, while the Gamma distribution serves as an acceptable model for an inverted CCD with very low dark current. Both distributions are greatly superior to the Gaussian distribution in this application.

Even though the control parameters are difficult to fit, the simulation models can still serve as effective sources of synthetic dark current data. They may provide the best solution for image processing pipeline algorithm development.

\section{Simulations}

Computer simulations were used to evaluate various hypotheses about the spatial distribution of defects and the generation rate distribution of these defects. The Poisson case (uniform spatial distribution of defects with a single generation rate) is presented in the first subsection. In subsequent subsections, the following hypotheses are considered:

1) The generation rates of defects are described by an additional random distribution.

2) The generation rate of defects is spatially non-uniform because of field enhancement.

3) The distribution of defects is non-uniform. 
In each section the methodology that was used to perform the simulation is described, followed by simulation results and discussion.

The first hypothesis is shown to be inadequate. The experimental observed dark current distribution can not be explained by any distribution of generation rates. The distributions produced by the second and third hypotheses are consistent with experimental observations. Although the field enhancement hypothesis has the strongest physical basis, it does not yield an analytical form that would be useful for characterization and simulation. The non-uniform defect hypothesis does not have a strong basis, but it suggests a useful empirical model.

\subsection{Uniform spatial distribution, single generation rate (Poisson distribution)}

If the defects were uniformly distributed and all shared a common generation rate, a Poisson distribution ${ }^{7}$ of dark current would result.

$$
P(x)=\frac{a^{x} e^{-a}}{x !} \quad x=0,1,2, \ldots \quad \text { Poisson distribution }
$$

\subsubsection{Results}

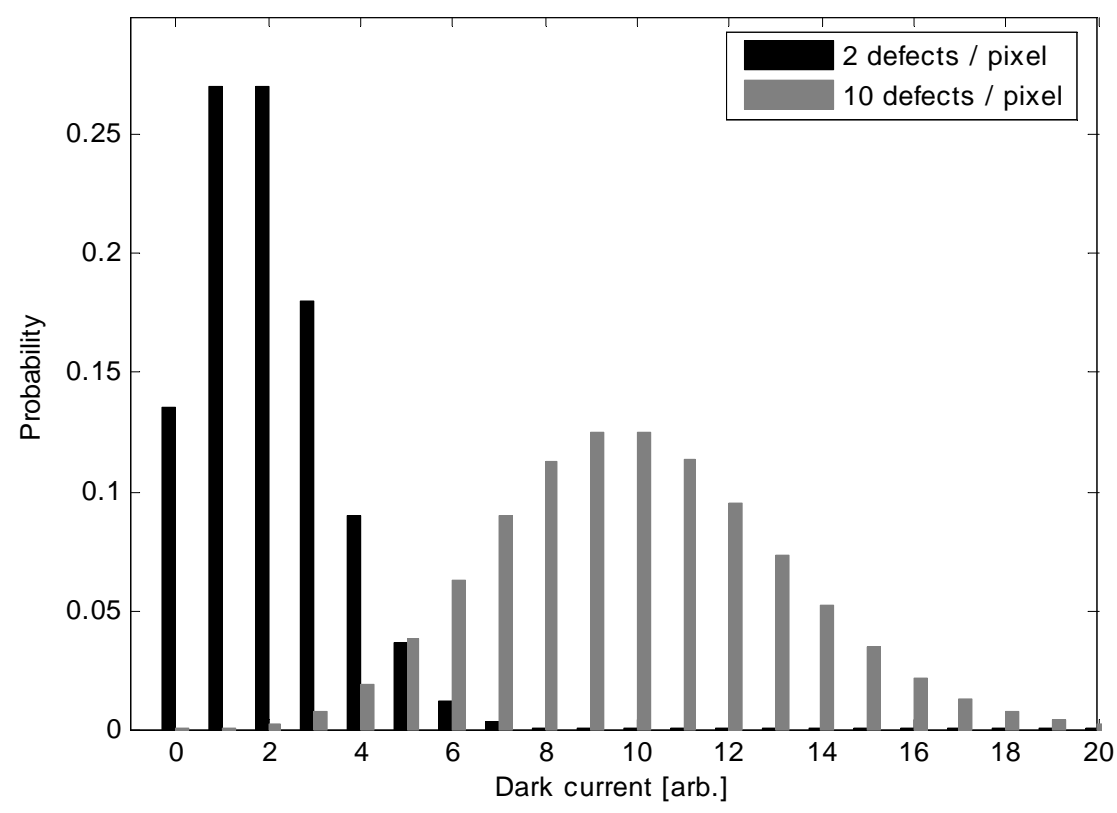

Figure 2: Poisson distribution, 3 defects per pixel and 10 defects per pixel (average)

\subsubsection{Discussion}

The Poisson distribution shown in Figure 2 is discrete. Although it is positively skewed when the number of defects per pixel is small, the skew disappears when the number of defects is large and the shape of the Poisson distribution approaches the shape of the Gaussian (Normal) distribution.

The SiO2/Silicon surface state density is about $10^{\wedge} 9 / \mathrm{cm}^{\wedge} 2$, or $15 / \mathrm{um}^{\wedge} 2$ [see ref. \#1]. Consequently hundreds of defects would be expected in a CCD or CMOS surface photodiode or photogate. The large surface hole populations in buried photodiodes and inverted CCDs fill the surface traps, reducing their effect. However the total number of active traps (surface and bulk) must still be large (>10), or a significant number of pixels with zero dark current would be observed in experimental measurements. 
Defects outside the photodiode that spread their charge to many pixels by diffusion would eliminate the discrete character of the distribution. However there is no physical mechanism for this kind of behavior. The Shockley-HallRead equation predicts that charge generation outside of the depletion region (between pixels) should be exceedingly small because of the high free carrier density.

The Poisson distribution is not consistent with experimental observations. The distribution associated with a large number of defects per pixel would not be skewed, and the experimental distribution does not appear to be discrete.

\subsection{Distribution of generation rates}

In the preceding case, all defects were assumed to share a common generation rate. It is more realistic to assume that a distribution of generation rates exists. If the distribution was broad enough, it might blend the discrete peaks of the Poisson distribution together and allow a continuous, positively skewed, distribution to emerge when the defect density is low. Alternatively, an asymmetric generation rate distribution might lead to a skewed distribution when the defect density is high. These two propositions are examined in this subsection.

\subsubsection{Methodology}

A "fair" Roulette algorithm was used to simulate the effect of distributed generation rates in Matlab. Initially an empty pixel array $\mathrm{p}[\mathrm{i}]$ of length $\mathrm{N}$ was created (e.g. the Roulette wheel). Subsequently an array of $\mathrm{N}^{*} \mu$ uniformly distributed random defect coordinate values $x[i, \mu]$ were created, where $\mu$ is the average number of defects per pixel (e.g. $N^{*} \mu$ is the number of Roulette balls). Another array of random variables $g\left[i, \mu_{\mathrm{G}}, \sigma_{\mathrm{G}}\right]$ of the same length was created to represent the generation rate distribution, according to the particular distribution that was used in the simulation (Gaussian or exponential). The generation rate distribution was characterized by parameters $\mu_{\mathrm{G}}$ and $\sigma_{\mathrm{G}}$. Iteration was used to examine every element $\mathrm{x}$ of the defect position array. The pixels array elements at those locations (determined by floor(x[i])) were incremented by g[i]. The process was repeated to include more than one type of defect in the simulation. The resulting probability distributions were obtained by computing the histograms of the pixel array values.

\subsubsection{Results}

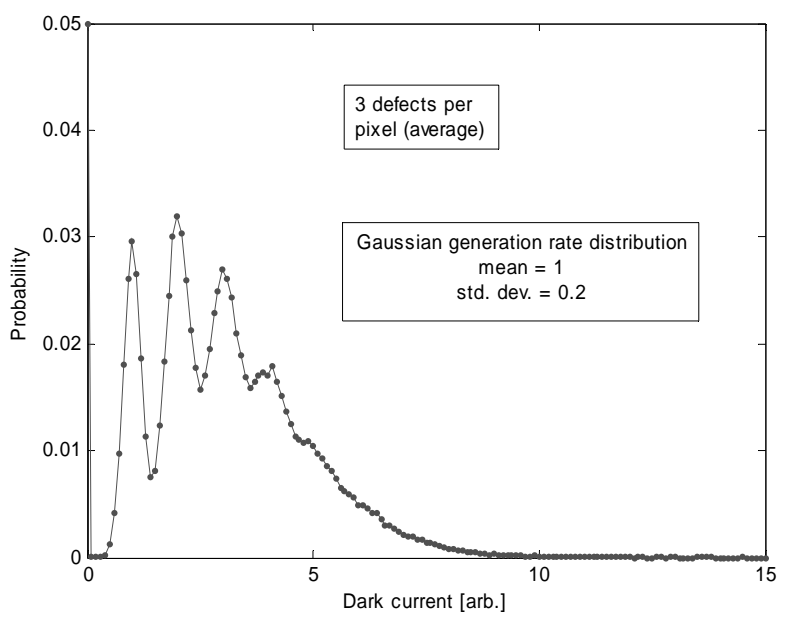

Figure 3: Gaussian distribution of generation rates, generation rate standard deviation $=0.2$

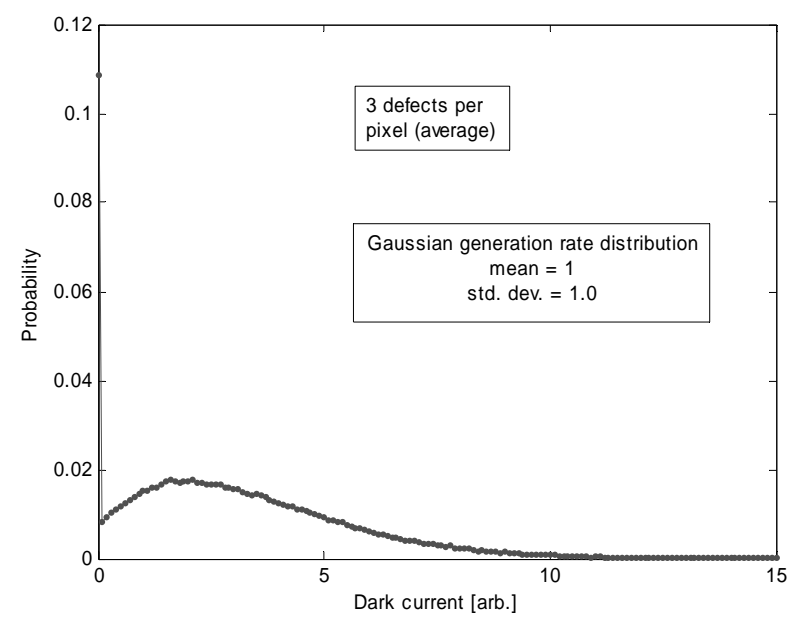

Figure 4: Gaussian distribution of generation rates, generation rate standard deviation $=1.0$

Figures 3 and 4 resulted from simulations that utilized a Gaussian distribution of generation rates. In both cases, a small value of only three average defects per pixel was assumed. When the standard deviation of the generation rate distribution is small, the underlying peaks of the Poisson distribution are clearly visible. When the standard deviation is large, the upper peaks of the Poisson distribution are smeared together. However a distinct discrete peak at zero dark current remains in both cases. 


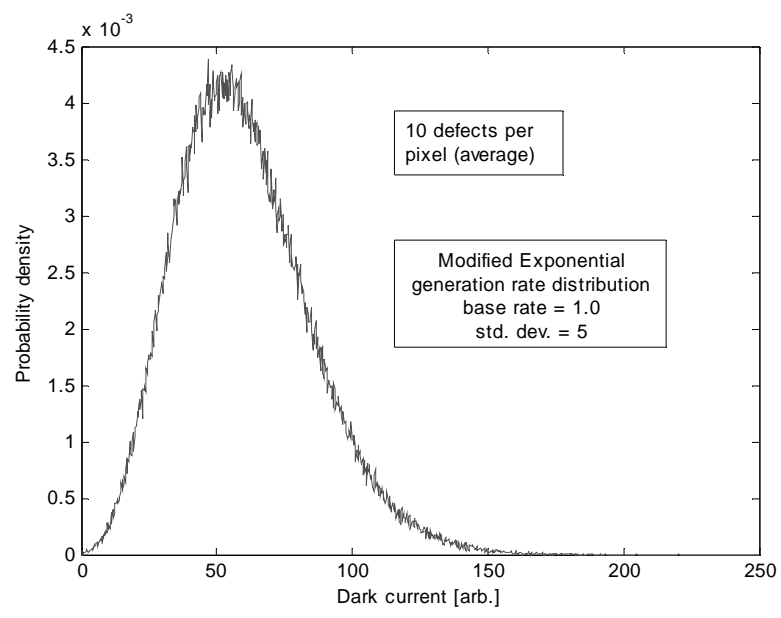

Figure 5: Exponential distribution of generation rates, 10 defects per pixel

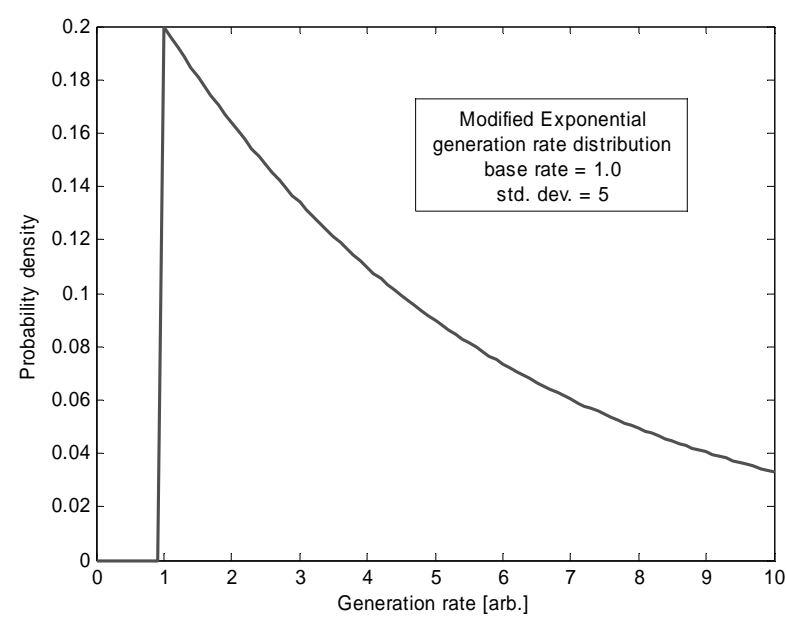

Figure 6: Exponential generation rate distribution used in simulation

Figure 5 resulted from a simulation in which a highly asymmetric exponential generation rate distribution was used. The generation rate distribution is shown in Figure 6. A moderate average number (10) of defects per pixel was assumed, which had the benefit of eliminating the discrete peak at zero dark current. However the distribution that resulted wasn't significantly skewed.

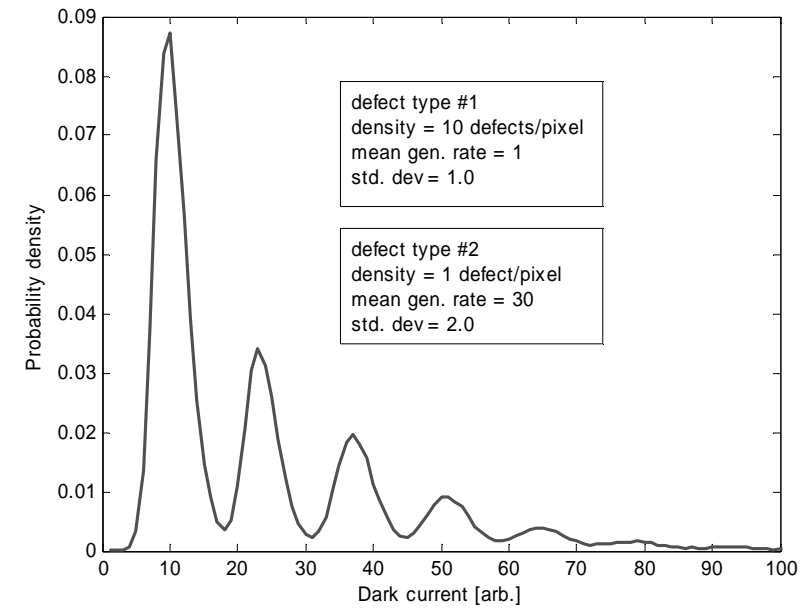

Figure 7: Gaussian distribution of generation rates, with two distinct mechanisms

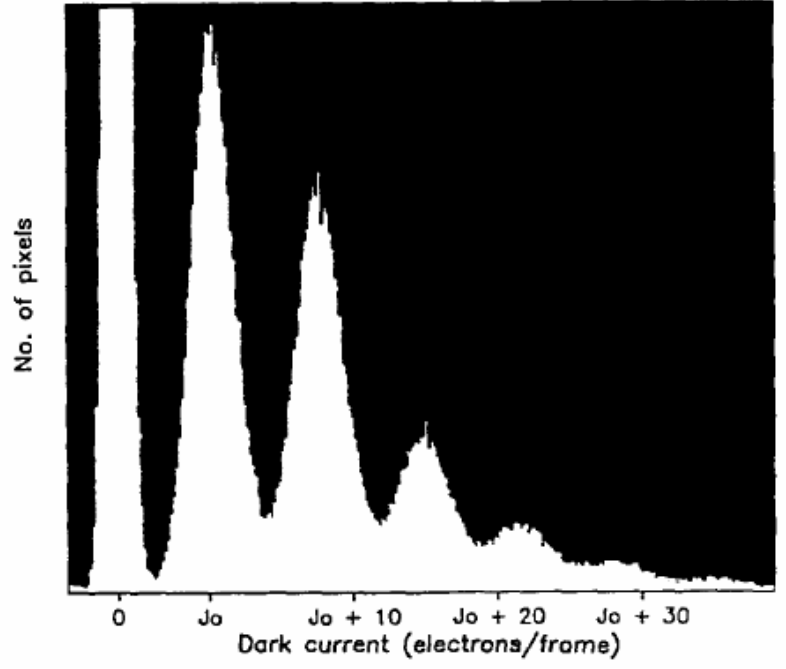

Figure 8: Experimental dark current distribution with deep-level traps [from ref. \#8 ].

Two distinct types of defects were modeled in the simulation that resulted in Figure 7. A high density of 10 defects per pixel was assumed for the defect with an average generation rate of one. A low density of one defect per pixel was assumed for the defect with an average generation rate of 30 (representing a metal ion impurity). A Gaussian distribution of generation rates was assumed in both cases. The distinct Poisson peaks of the high generation rate species are clearly visible. The simulation results are very similar to the experimental histogram in Figure 8 , from reference 8 .

\subsubsection{Discussion}

If the average number of defects per pixel is small, the discrete nature of the underlying Poisson distribution can not be concealed by the application of a generation rate distribution. A broad generation rate distribution can only spread the 
positive peaks; it can not conceal the significant peak at zero dark current (which is not observed experimentally). The appearance of discrete peaks in the experimental distribution of Figure 8 substantiates this hypothesis.

If the average number of defects per pixel is large, the central limit theorem comes into force. The net distribution is obtained by convolving the generation rate distribution against itself " $n$ " times for each peak in the Poisson distribution and then summing the weighted results. The final distribution approaches a Gaussian, irrespective of the original shape of the generation rate distribution.

Experimentally observed distributions are continuous and positively skewed. Therefore they can not be explained by defects that are uniformly distributed in position, irrespective of the distribution of generation rates. Some sort of spatial non-uniformity is required. Either the defects behave differently based on spatial location, or the distribution of defects is non-uniform. These hypotheses will be examined in the next two subsections.

\subsection{Spatial variation of field enhancement}

The Poole-Frenkel effect describes the increase in emission rate from a defect in the presence of an electric field ${ }^{9}$. Because the electric field varies across the photodiode (or potential well, in the case of a CCD), the charge generation from some defects is enhanced more than others. In order to calculate the distribution resulting from this effect, one would need to know the field profile inside the pixel (presumable from a MEDICI simulation) and the defect distribution density in the bulk and on the surfaces. This kind of calculation has been performed for proton-irradiated CMOS APS sensors resulting in accurate estimation of the dark current distribution ${ }^{10}$. The same mechanism must apply to defects that are not the result of proton irradiation, and is likely to be the explanation for the skewed dark current distribution.

\subsubsection{Methodology}

The Roulette method described in the previous section was modified slightly to accommodate field enhancement. The degree of field enhancement is determined by the fractional position of the defect within the 1-D pixel, as shown in Figure 9. The field enhancement is described as a function of the fractional position, $\Delta x(=x[i]-f l o o r(x[i]))$. In these simulations the function $\mathrm{g}_{\mathrm{FE}}(\Delta \mathrm{x})=1+\mathrm{a} \exp (\mathrm{b} * \Delta \mathrm{x}) / \exp (\mathrm{b})$ was arbitrarily chosen to describe the gain due to field enhancement. Parameter "a" controls the maximum gain due to field enhancement, while parameter "b" controls the distribution of field enhancement across the pixel. The shape of this function is unimportant: all that matters is its histogram. Since the cumulative probability for electric field in the volume of the pixel falls of rapidly with electric field strength, this provides a reasonable model. A Gaussian distribution of generation rates was also included in the simulation, with a mean value of one and a standard deviation of 0.3 . In this case the pixel elements at the locations determined by floor $(\mathrm{x}[\mathrm{i}]))$ were incremented by $\mathrm{g}_{\mathrm{FE}}(\Delta \mathrm{x}[\mathrm{i}]) * \mathrm{~g}[\mathrm{i}]$.
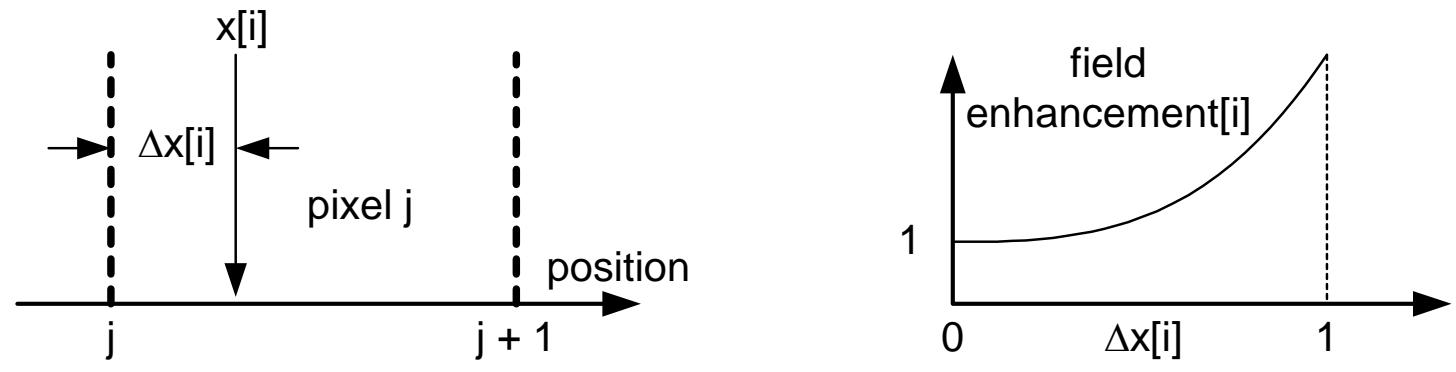

Figure 9: Field enhancement method

\subsubsection{Results}




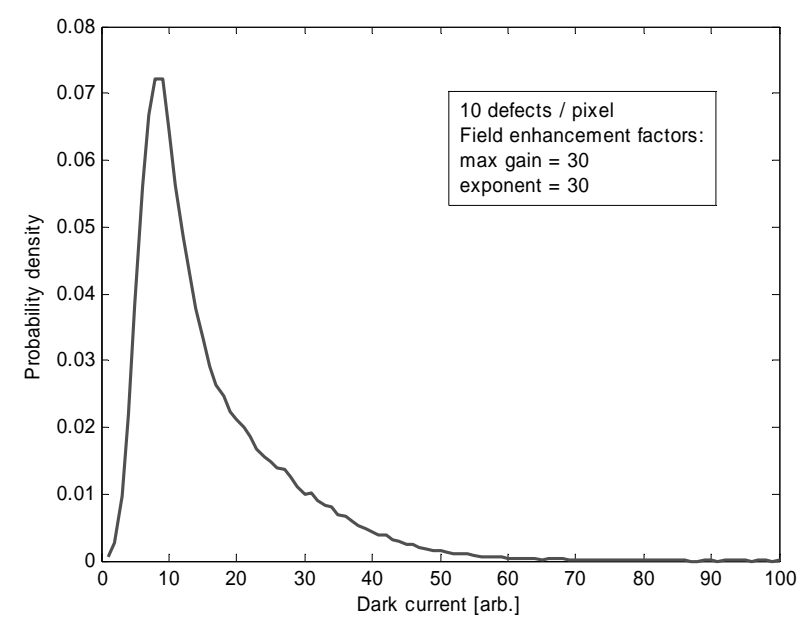

Figure 10: Exponential variation of field enhancement, 10 defects per pixel

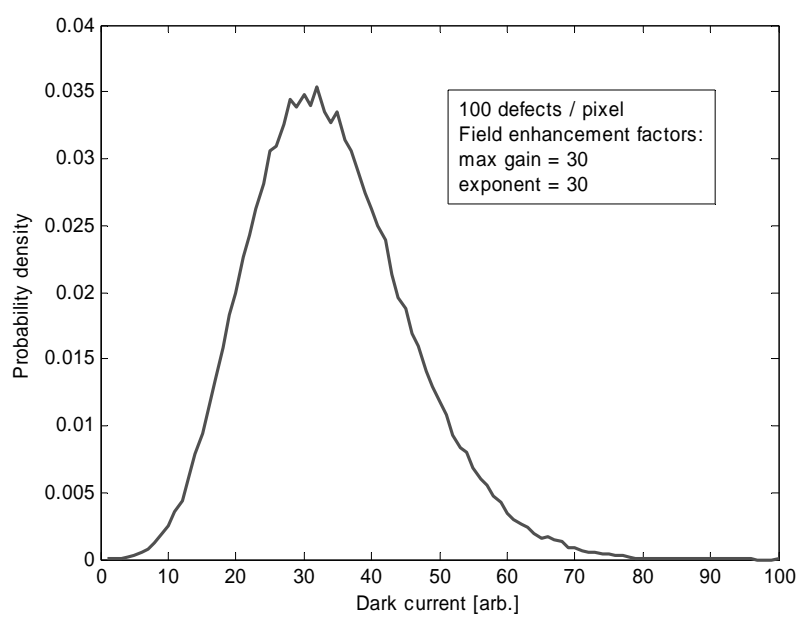

Figure 11: Exponential variation of field enhancement, 100 defects per pixel

The histograms in Figures 10 and 11 resulted from simulating the effect of field enhancement with different parameters. In both cases, the maximum field enhancement gain is high, but the fraction of the pixel that provides significant field enhancement is small. By varying the parameters it is possible to produce a wide range of different positively skewed distributions. When the defect density is very high, the intrinsic variance of the defect distribution is so high that it swamps out the variable gain effect, and a Gaussian-like distribution results (as shown in Figure 10). A wide range of positively skewed single-peaked distributions can be generated with this method. However the choice of parameters is critical.

\subsubsection{Discussion}

Since the field enhancement model has a strong physical basis, an associated analytical distribution would be useful for characterization and simulation. An analytical derivation can be approached by dividing each 1-D pixel into a number of discrete sections. The number of defects in each section would be described by a Poisson-distributed random variable $x_{i}$ with distribution $\mathrm{P}_{\mathrm{x}}\left(x_{i}\right)$. The number of defects would be multiplied by the field enhancement gain, $a_{i}$, for that section, resulting in a new random variable $y_{i}$, with distribution $\left(1 / a_{i}\right) \operatorname{Px}\left(y_{i} / a_{i}\right)$. The sum of the $y_{i}$ would represent the dark current of the pixel. Therefore the dark current distribution would be represented by the convolution of the distributions of all of the $y_{i}$. The problem can be transformed into one of multiplication in the Fourier domain, but a simple representation for the product of a number of scaled distributions did not present itself to the author. However the model is still useful for generating synthetic dark current, given the correct empirically-derived parameters.

\subsection{Non-uniform spatial distribution}

In all of the previous cases, the spatial distribution of defects was assumed to be uniform. In this case a non-uniform spatial distribution is considered. The non-uniform distribution represents the clustering of defects. This might occur if some defects tended to attract (e.g. getter) other defects.

\subsubsection{Methodology}

The non-uniform spatial distribution of defects was simulated by using an "unfair" Roulette algorithm. In the unfair algorithm, the width of each Roulette bin grows with the number of balls (defects) that it contains, as shown in Figure 12. The "attraction" (bin width) function is chosen arbitrarily. In this case an attraction function of $w=1+a^{*} n$ was used. A separate array was used to store the positions of the bin boundaries. The positions are updated after every "spin" of the wheel. The length of the uniform defect position distribution is increased after each spin to accommodate the growing "circumference" of the wheel. In order to simplify the calculation, the distribution of generation rates was ignored. Based upon previous results, the number of defects per pixel was assumed to be large. 


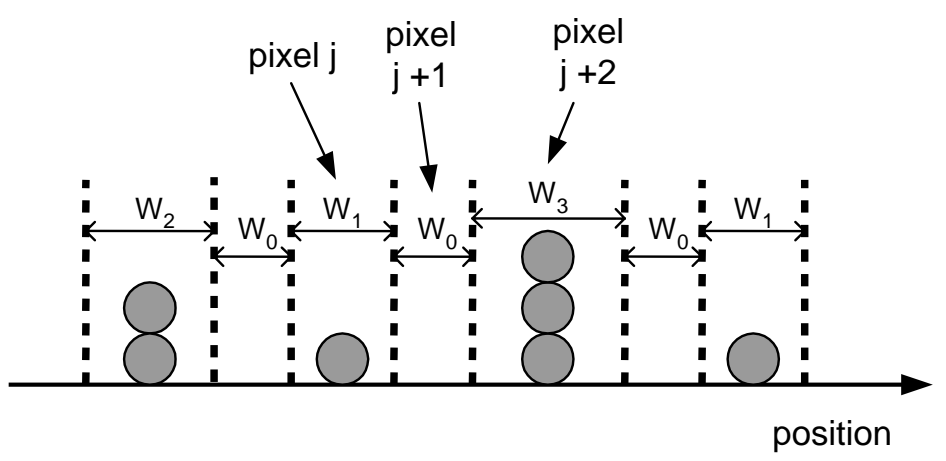

Figure 12: Unfair "Roulette” method

\subsubsection{Results}

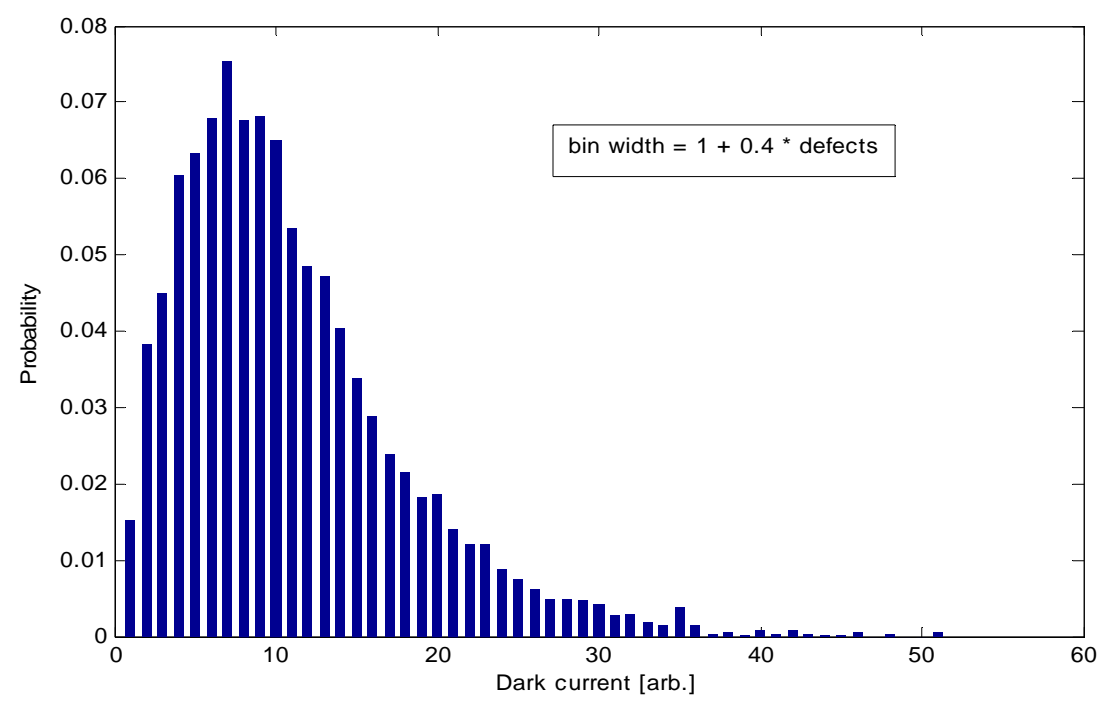

Figure 13: Histogram resulting from non-uniform spatial distribution of pixels

The histogram in Figure 13 resulted from the non-uniform spatial distribution of pixels, with a linear "attraction" factor of 0.4. A value of ten was chosen for the mean number of defects per pixel. As shown previously, a discrete peak at zero dark current becomes apparent when the mean defect number is small. When the "attraction" factor is decreased to 0.1, the distribution becomes almost Gaussian. When it is increased to 1.0, it becomes almost exponential. The discreteness of the distribution is visible because the underlying generation rate distribution has been neglected. As with the previous case, a wide range of distribution shapes can be produced by this method.

\subsubsection{Discussion}

Even though this model is conceptually simpler than the previous one, it is computationally the least efficient. The recalculation of the bin widths at each step prevents the execution from being effectively parallelized or pipelined. The model is capable of producing highly skewed distributions, even when the average defect density is high. However the choice of the attraction function is entirely arbitrary and no physical basis for this behavior has been reported.

Another approach to producing a non-uniform distribution would be to first create a uniform distribution and then to operate on the resulting values to further increase the defect count in pixels which already have high defect counts. An operation that has this characteristic is exponentiation. However the exponentiation of a Gaussian random variable results in the familiar Log-Normal distribution, which is considered as an empirical model in the next section. 


\section{Empirical models}

Although the simulations have provided physical insight into the potential causes the skewed dark current distribution, they have not yielded an analytical model that is suitable for characterization and simulation. Consequently an empirical model is required. The ideal empirical model would have the following characteristics:

1) It would have a simple analytical representation

2) It would posses only a few somewhat orthogonal control parameters (e.g. scale and shape)

3) The optimum control parameters could be easily determined from the experimental data

4) A simple and efficient method for simulating the distribution would exist.

Many common single-peaked positive distributions don’t fit the experimental data very well, including the Normal, Maxwell, Rayleigh and Weibull distributions. Three that "baer" closer examination are the Log-Normal, Gamma and Inverse Gamma distributions.

\subsection{The Log-Normal PDF}

The Log-Normal distribution ${ }^{11}$ is suggested by the non-uniform defect density simulation model. If a Gaussian distribution results from distributing defects uniformly, then perhaps a non-uniform distribution can be created by "pulling" on the tail of a Gaussian. This is exactly what the Log-Normal distribution does: the values are exponentiated, "stretching" the tail of the Gaussian distribution much more than the peak. Other related distributions that used polynomial "stretching" functions were investigated, but they did not produce realistic distributions. The Log-Normal PDF is:

$$
\operatorname{PLN}(x ; \mu, \sigma)=\frac{1}{x \sigma \sqrt{2 \pi}} e^{\frac{-(\ln (x)-\mu)^{2}}{2 \sigma^{2}}} \quad \text { Log }- \text { Normal PDF }
$$

Although the expressions for the mean and variance of the Log-Normal distribution are complicated, the parameters of the distribution can easily be obtained by taking the logarithm of the experimental data. The parameters $\mu$ and $\sigma$ are simply the mean and standard deviation of the log data.

Simulation of the Log-Normal distribution is also very simple. An array of normally distributed random variables is generated and then simply exponentiated.

\subsection{The Gamma PDF}

The Gamma $\mathrm{PDF}^{12}$ is a continuous generalization of the discrete Poisson distribution. The Gamma PDF is:

$$
\mathrm{P}_{\mathrm{G}}(x ; k, \theta)=x^{k-1} \frac{e^{-x / \theta}}{\theta^{k} \Gamma(k)} \quad \text { Gamma PDF }
$$

The mean of the Gamma PDF is $(k \theta)$, while the variance is $\left(k \theta^{\wedge} 2\right)$. These expressions can be inverted to yield the parameters of the distribution in terms of the mean and variance of an experimental distribution:

$$
\theta=\text { variance } / \text { mean; } k=\text { mean }^{2} / \text { variance }
$$

Methods for generating random variables with the Gamma distribution are well known, and available in the MATLAB statistics toolkit and in the Mathematica continuous distribution statistics package.

\subsection{The Inverse Gamma PDF}

The Inverse Gamma $\mathrm{PDF}^{13}$ is the distribution of the inverse of the Gamma random variable. The Inverse Gamma PDF is:

$$
\operatorname{PIG}(x ; \alpha, \beta)=\frac{\beta^{\alpha}}{\Gamma(\alpha)} x^{-(\alpha+1)} e^{-\beta / x} \quad \text { Inverse Gamma PDF }
$$


The mean of the Inverse Gamma distribution is $\beta /(\alpha-1)$ (for $\alpha>1$ ) and the variance is $\beta^{2} /\left((\alpha-1)^{2}(\alpha-2)\right.$ ) (for $\left.\alpha>2\right)$. These equations must be solved numerically to determine $\alpha$ and $\beta$ from the parameters of the experimental distribution.

Methods for generating random variables with the Inverse Gamma distribution are known, but are not widely accessible.

\subsection{Fitting experimental data to the model}

The fits of the three empirical distributions to the dark current histogram of a 3T CMOS APS sensor are shown in Figure 14. In this case the second order statistics of the experimental distribution were used to determine the best fit parameters. The parameters used were: Gamma $\{k=3.56, \theta=4.31\}$, Inverse Gamma $\{\alpha=5.56, \beta=69.9\}$, and LogNormal $\{\mu=2.61, \sigma=0.498\}$. Although the Log-Normal distribution matches the experimental data very well, it is hard to attach any physical significance to the result. The Log-Normal distribution usually applies to the product of a large number of independent random variables. There is no related "product" mechanism in an image sensor.

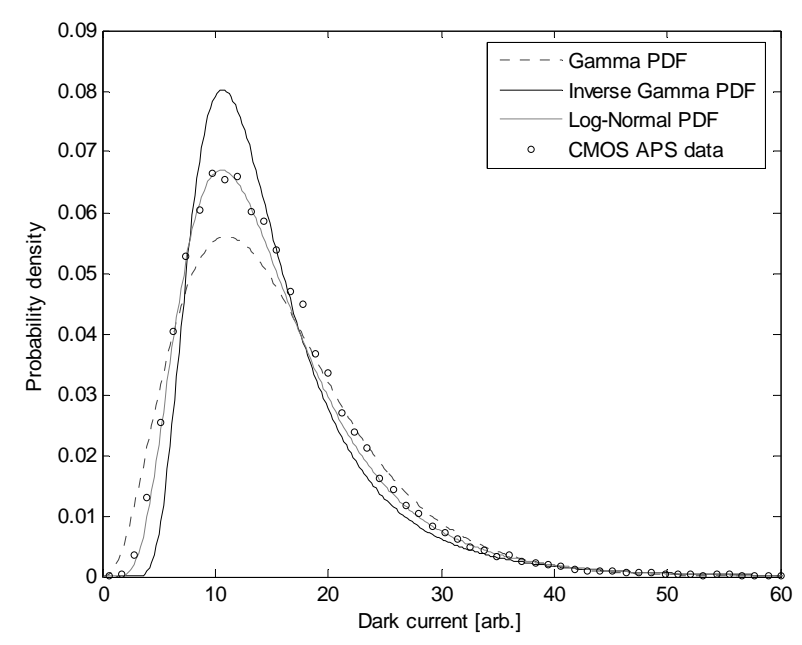

Figure 14: Comparison of Log-Normal PDF with 3T CMOS APS histogram

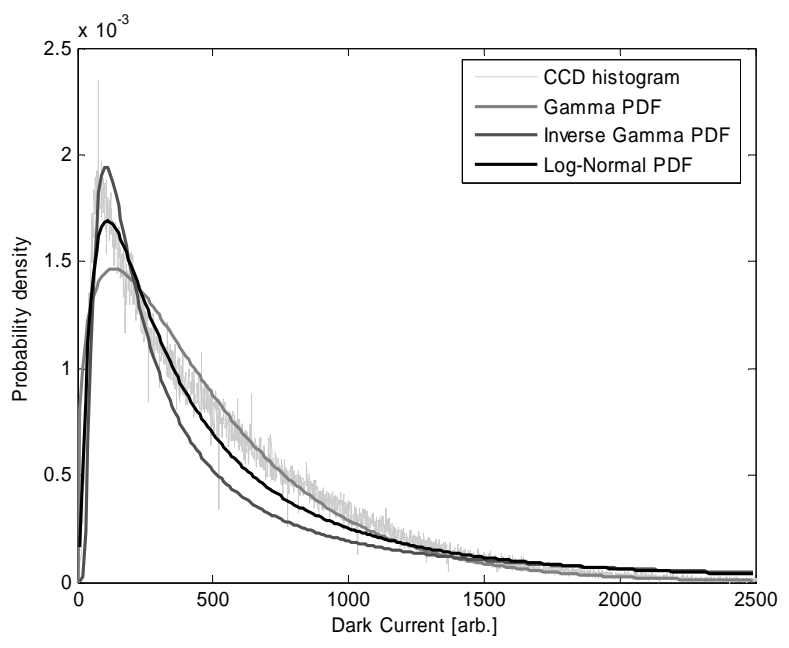

Figure 15: Comparison of Log-Normal PDF with inverted CCD histogram [data courtesy of Dalsa]

The fits of the three distributions to the dark current histogram of an inverted CCD are shown in Figure 15. In this case non-linear curve fitting was used to obtain the fitting parameters (parameters obtained from the second order statistics of the experimental distribution produced poor fits). The parameters used were: Gamma $\{k=1.36, \theta=370\}$, Inverse Gamma $\{\alpha=0.698, \beta=177.6\}$, and Log-Normal $\{\mu=5.98, \sigma=1.123\}$. The Gamma distribution provided the best match. The Inverse Gamma and Log-Normal distributions did not decay rapidly enough at high dark current levels. All three functions provide a much better fit than the Normal PDF does.

The best fit of the field enhancement simulation model to the dark current histogram of the inverted CCD used in Figure 15 is shown in Figure 16. A scale factor was added to the model to account for the charge to voltage conversion ratio, electronic amplification and digital scaling. In this case the parameters were obtained by trial and error. The parameters were: \{average defect density $=3$ / pixel, maximum field enhancement $=15$, exponent $=8$, generation rate standard deviation $=0.35$, scale factor $=50\}$. The maximum field enhancement value of 15 is consistent with the field strengths reported in reference \#10. An accurate fit could not be obtained when a higher average defect density was used. As a consequence, the distribution resulting from the simulation has a significant discrete peak at zero dark current which is not visible in the figure. The physical significance is unclear. These zero values could be randomly replaced with positive values from other pixels without changing the shape of the distribution. This method could be used to create synthetic dark current images that could be used in the development of image processing algorithms. 


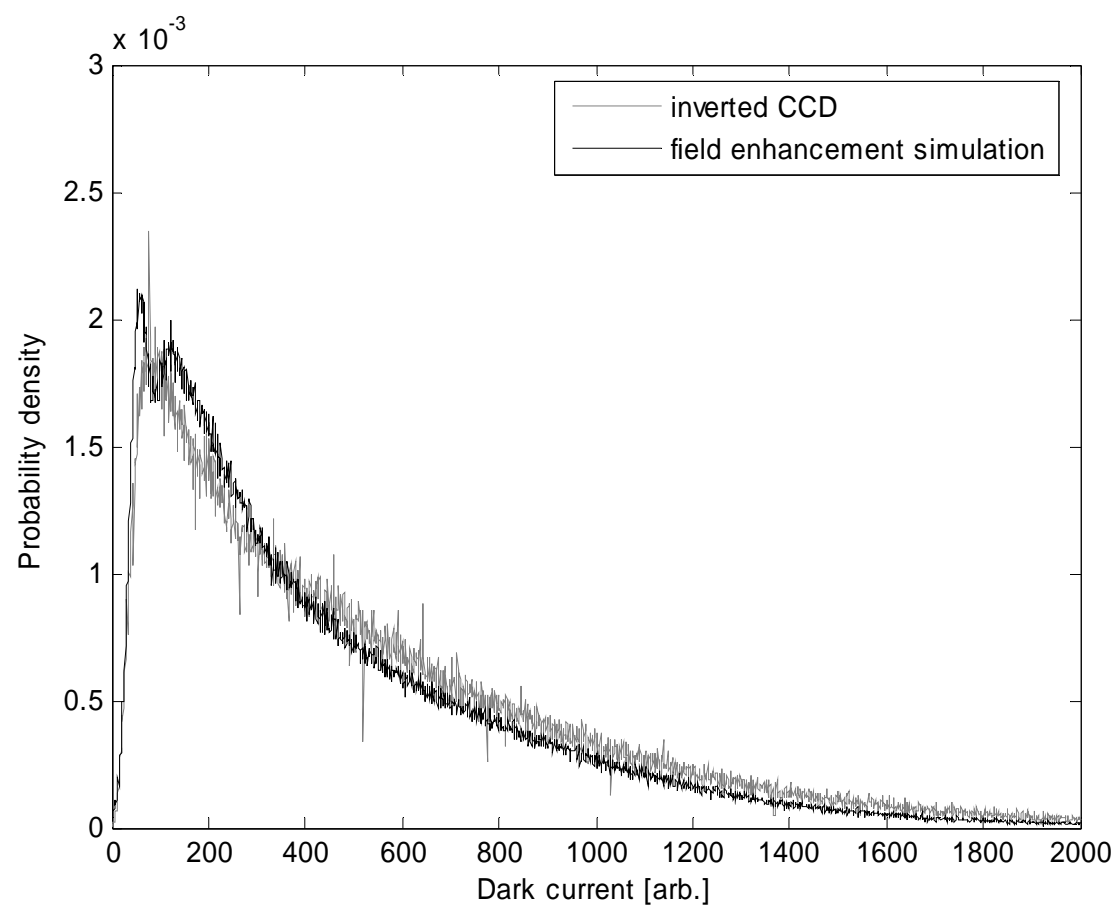

Figure 16: Comparison of field enhancement simulation with inverted CCD histogram.

\subsection{Discussion}

The best empirical distribution for dark current characterization and simulation is dependent on the image sensor. The Log-Normal distribution works very well for conventional 3T APS CMOS sensors with comparatively high dark current. The Gamma distribution works reasonably well for inverted CCD sensors with low dark current. When these analytical distributions don't fit well enough, a simulation model can be used instead. All of these solutions provide much more accurate characterization and simulation than the commonly used Gaussian model.

\section{Conclusions}

A number of different potential explanations for the positive skew observed in experimental dark current distributions have been examined by computer simulation. A number of important conclusions can be drawn from the simulation results:

1. When the average number of defects per pixels is low $(<5)$, the probability that many pixels will contain no defects becomes significant. Since a spike is not observed in empirical dark current distributions at zero dark current, the number of defects per pixel must be large. Alternatively, the experimental data may be masked by temporal noise.

2. A distribution of generation rates is not sufficient to explain positively skewed experimental distributions. When the average defect density is low, the generation rate distribution is incapable of masking the discrete nature of the underlying Poisson distribution. When the average defect density is high, the central limit theorem causes the resulting dark current distribution to approach a Gaussian, irrespective of the shape of the generation rate distribution.

3. Some kind of spatial inhomogeneity is required to explain the experimental distributions. The inhomogeneity can appear as non-uniform gain applied to uniformly distributed defects, or as non-uniformly distributed defects themselves. The Poole-Frenkel field-induced barrier lowering effect provides a physical mechanism for the former, while no physical mechanism has been reported for the latter. 
Several different positively-skewed distributions have been proposed as empirical replacements for the Gaussian distribution in characterization and simulation. Of these, the Log-Normal distribution provides the best results. Its second order statistics can be used to easily fit the distribution to data from 3T APS CMOS sensors. It also provides a simple simulation model, since synthetic dark current images can be generated by exponentiating images created by Gaussian random number generators. However the Log-Normal distribution did not fit the experimental data from an advanced inverted CCD very well. The Gamma distribution produced an acceptable fit in this case. When these distributions are inadequate, the field enhancement simulation model can be used to obtain a closer fit.

Other important characteristics of dark current, such as the temperature dependence ${ }^{14,15}$ and random telegraph behavior $^{16,17}$, are also begging for better characterization and simulation models. This presents a fruitful avenue for future research.

\section{Acknowledgments}

I would like to acknowledge Boyd Fowler and Albert Theuwissen for their helpful advice. I would additionally like to thank Albert for providing the inverted CCD histogram.

\section{References}

1. J.R. Janesick, Scientific Charge-Coupled Devices, SPIE Press, Bellingham, WA (2001).

2. A.J.P. Theuwissen, Solid-State Imaging with Charge-Coupled Devices, Kluwer Academic Publishers, Dordrecht, The Netherlands (1995).

3. R.D. McGrath, J. Doty, G. Lupino, G. Ricker and J. Vallerga, "Counting of deep-level traps using a charge coupled device”, IEEE Trans. on Electronic Devices, Vol. ED-34, No. 12, pp. 2555-2557 (Dec. 1987).

4. G.A. Hawkins, E.A. Trabka, R.L. Nielsen and B.C. Burkey, "Characterization of generation currents in solid-state imagers”, IEEE Trans. on Electron Devices, Vol. ED-32, No. 9, pp. 1806-1816 (Sept. 1985).

5. D. Donahue, “A model for random pixel clustering in large format CCD's”, Proceedings of the SPIE, Vol. 1900, pp. 85-90 (1993).

6. J.C. Mullikin, L.J. van Vliet, H. Netten, F.R. Boddeke, G. van der Feltz and I.T. Young, "Methods for CCD camera characterization”, Proc. SPIE, Vol. 2173, pp. 73-84 (1994).

7. A.W. Drake, Fundamentals of Applied Probability Theory, McGraw-Hill, New York (1967).

8. W.C. McColgin, J.P. Lavine, J. Kyan, D.N. Nichols, and C.V. Stancampiano, "Dark current quantization in CCD image sensors", Proc. IEDM, pp. 113-116 (1992).

9. J.T. Bosiers, E. Roks, H. Peek, A.C. Kleimann and A.G. van der Sijde, “An S-VHS compatible 1/3"' color FT-CCD imager with low dark current by surface pinning”, IEEE Transactions on Electronic Devices, Vol. 42, No. 8, pp. 1449-1460 (Aug. 1995).

10. J. Bogaerts, B. Dierickx and R. Mertens, "Enhanced dark current generation in proton-irradiated CMOS active pixel sensors”, IEEE Trans. on Nuclear Science, Vol. 49, No. 3, pp1513-1521 (June 2002).

11. Wikipedia contributors, "Log-normal distribution," Wikipedia, The Free Encyclopedia, http://en.wikipedia.org/w/index.php?title=Log-normal_distribution\&oldid=28636728 (accessed December 12, 2005).

12. Wikipedia contributors, "Gamma distribution," Wikipedia, The Free Encyclopedia, http://en.wikipedia.org/w/index.php?title=Gamma_distribution\&oldid=30918236 (accessed December 12, 2005).

13. Wikipedia contributors, "Inverse-gamma distribution," Wikipedia, The Free Encyclopedia, http://en.wikipedia.org/w/index.php?title=Inverse-gamma_distribution\&oldid=23858012 (accessed December 12, 2005).

14. R. Wildenhorn, L. Munderman, A. Rest and E. Bodegom, "Meyer-Neldel rule for dark current in charge-coupled devices", Journal of Applied Physics, Vol. 89, No. 12, pp. 8179-8182 (June 2001).

15. R. Widenhorn, M.M. Blouke, A. Weber, A. Rest and E. Bodegom, “Temperature dependence of dark current in a CCD”, Proc. SPIE, Vol. 4669, pp. 193-201 (2002).

16. J. Bogaerts, B. Dierickx and R. Mertens, "Random telegraph signals in a radiation-hardened CMOS active pixel sensor”, IEEE Trans. on Nuclear Science, Vol. 49, No. 1, pp. 249-257 (Feb. 2002).

17. H. Hopkins and G.R. Hopkinson, "Random telegraph signals from proton-irradiated CCDs", IEEE Trans. on Nuclear Science, Vol. 40, No. 6, pp. 1567-1574 (Dec. 1993). 